\section{NPM1 mutation is not associated with prolonged complete remission in acute myeloid leukemia patients treated with hypomethylating agents}

Acute myeloid leukemia (AML) is a genetically heterogeneous group of neoplasms with different prognostic molecular biomarkers. In the 2016 revision of myeloid malignancies, the World Health Organization classified AML with mutated nucleophosmin (NPM1) as a distinct entity due to its unique biological and clinical profiles. ${ }^{1}$

Mutated NPM1 is seen in $25-30 \%$ of cases of AML and its incidence does not decrease with older age. ${ }^{2}$ While an NPM1 mutation is associated with better prognosis in AML patients treated with intensive chemotherapy, it is unknown whether it also confers a good prognosis to patients unfit for intensive chemotherapy who are treated with hypomethylating agents.

To address this issue, we retrospectively collected and analyzed data from 71 mutation-positive AML patients treated with hypomethylating agents between 2007 and 2016 in eight European centers and one American center. The diagnosis of AML was reviewed according to the 2016 World Health Organization revision of myeloid neoplasms and acute leukemia, and cytogenetic risk was stratified according to the Southwest Oncology Group criteria. ${ }^{1,4}$ Molecular analysis of NPM1 and FLT3 status was performed in all patients at diagnosis. Minimal residual disease was determined by real-time quantitative polymerase chain reaction analysis. Response to treatment and relapse were assessed according to International Working Group criteria. ${ }^{5}$ Comparisons of categorical and continuous variables were analyzed by the $\chi^{2}$ /Fisher exact test and Mann-Whitney $U$ test, respectively. Overall survival was measured from the time of starting hypomethylating agent treatment to the date of death or censored at the date of the last followup. Survival estimates were calculated using the KaplanMeier method and compared with the log-rank test. This research was carried out in accordance with institutional ethics committee guidelines and with the Declaration of Helsinki.

Thirty-four patients received upfront treatment with hypomethylating agents. These patients had been considered unfit for intensive chemotherapy based on age (>80 years old), cardiac disorders or other comorbidities, and according to each institution's recommendations. Their median age was 77 years (range, 55-85) and the male-to-female ratio was 0.72 . Their median baseline white blood cell count was $8.2 \times 10^{9} / \mathrm{L}$ (range, $0.1 \times 10^{9} / \mathrm{L}$ $83 \times 10^{\circ} / \mathrm{L}$ ) and all but three had an intermediate karyotype according to the Southwest Oncology Group criteria: of the three patients without an intermediate karyotype, two had an unfavorable karyotype and cytogenetic analysis failed in the third. Twelve patients had an NPM1 type A mutation, four had a type B mutation, two had a type D mutation, and data were not available for 16 patients. Eighteen patients were tested for IDH mutations: one had an IDH1 R132H mutation; four had IDH2 mutations (2 R140Q, 1 R172S and 1 not available). Seven had an FLT3-ITD, and three had therapy-related AML. Seventeen patients received azacitidine, ten decitabine, and seven guadecitabine. The dose and administration schedule of the hypomethylating agents were generally those approved by the Food and Drug Administration or European Medicine Agency for azacitidine and decitabine, (sometimes reduced due to the patient's age or comorbidities), and $60 \mathrm{mg} / \mathrm{m}^{2} /$ day, 5 days every 4 weeks for guadecitabine. The median number of treat-
Table 1. Baseline characteristics of the studied groups treated upfront with hypomethylating agent.

\begin{tabular}{lcc} 
Median age & $\begin{array}{c}\text { NPM1 } \\
\text { cohort, } n=34\end{array}$ & $\begin{array}{c}\text { non-NPM1 } \\
\text { cohort, } n=92\end{array}$ \\
Male-to-female ratio & 76.9 & 74 \\
\hline Median WBC at HMA onset & 0.72 & 1.4 \\
Unfavorable cytogenetics & $8.2 \times 10^{9} / \mathrm{L}$ & $3.9 \times 10^{9} / \mathrm{L}$ \\
Intermediate cytogenetics & $5.8 \%$ & $64 \%$ \\
Median number of cycles & $91.2 \%$ & $28.1 \%$ \\
\hline Marrow blast count at HMA onset $\geq 30 \%$ & $87 \%$ & 7 \\
\hline WBC: white blood count; HMA: hypomethylating agent. &
\end{tabular}

ment cycles was eight (range, 1-18). The overall response rate was $45.5 \%$, given that eight patients $(23.5 \%)$ had a complete response, four patients $(12 \%)$ had a complete response with incomplete recovery of blood counts and three $(9 \%)$ had a partial response. The median overall survival was 280 days (Figure 1) and only one patient was alive after 2 years, but he eventually relapsed and died at 24.5 months. The median number of cycles to achieve the best response was five (range, 1-7). The overall response rates were $37.5 \%$ and $48 \%$ in patients with and without the FLT3-ITD, respectively $(P=0.69)$. No factors were seen to be prognostic of response or overall survival, including the type of hypomethylating agent given, FLT3 and IDH status and white blood cell count. Four of the six patients in whom NPM1 minimal residual disease in the bone marrow was assessed achieved a $>3$ log reduction after six cycles of treatment, but three of them relapsed within less than 6 months of this reduction, and the other one relapsed 16 months after the onset of treatment with a hypomethylating agent.

We compared patients of the present series with 92 NPM1-negative AML patients treated upfront with azacitidine between 2007 and 2012 by our group (Table 1). ${ }^{6}$ The median age of this cohort was 74 years (range, 44 88 ), $69 \%$ had unfavorable cytogenetics, and $63 \%$ had a TP53 mutation. Despite a very different molecular profile, there was no difference in overall survival between the NPM1-positive and -negative cohorts, suggesting a limited therapeutic impact of hypomethylating agents in NPM1-positive AML (median 280 versus 291 days, respectively; $P=0.53$ ) (Figure 1 ).

The remaining 37 patients of the present cohort received hypomethylating agents as the second or subsequent line of treatment. Their median age was 65 years (range, $36-87$ ), the male-to-female ratio was 0.7 , their median white blood cell count at the time of starting treatment with hypomethylating agents was $4.3 \times 10^{\circ} / \mathrm{L}$ (range, $0.4 \times 10^{9} / \mathrm{L}-50 \times 10^{\circ} / \mathrm{L}$ ), and 14 had FLT3-ITD. All patients had received previous anthracycline/cytarabinebased chemotherapy, seven had previously undergone allogeneic stem cell transplantation and one had had an autologous transplant. In this group, azacitidine was given to 30 patients, decitabine to seven patients and no patient received guadecitabine. The median number of treatment cycles was four (range, 1-14). The overall response rate was $24.5 \%$, with eight patients $(21.5 \%)$ achieving a complete response and one (3\%) having a complete response with incomplete recovery of blood counts. The median overall survival was 269 days. Two of the patients who achieved a complete response subsequently underwent allogeneic stem cell transplantation: 


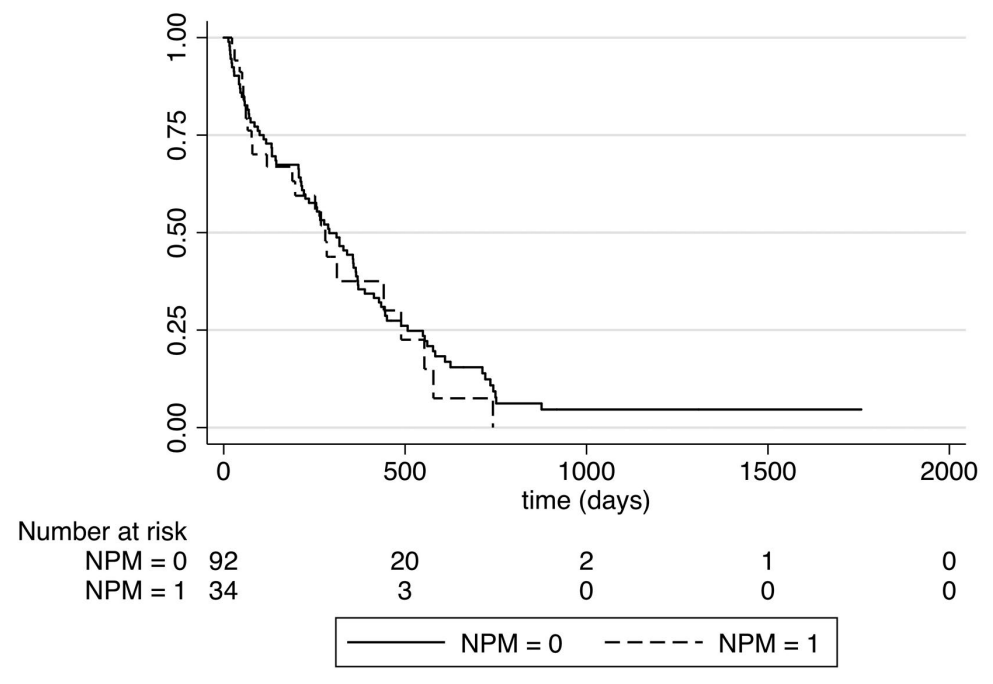

Figure 1. Overall survival after the start of hypomethylating agent treatment as firstline therapy. The dashed line represents patients of the present series with mutated NPM1. The solid line represents a historical control cohort of patients without NPM1 mutations (the majority of whom had a complex karyotype). one died of transplant complications, and one was alive in complete response 4 months after the transplant. Among the 14 patients with FLT3-ITD, 11 received concomitant therapy with sorafenib, with no impact on the overall response rate or overall survival, compared to those of patients with FLT3-ITD who did not receive sorafenib $(P=0.73)$. No prognostic factors for response or overall survival were seen, including the type of hypomethylating agent, FLT3 status, or white blood cell count.

Intensive chemotherapy can be curative in patients with NPM1-positive AML, including elderly ones: the 5year overall survival rate of a British cohort of NPM1-positive AML patients aged $\geq 60$ years treated with intensive chemotherapy was $60 \%{ }^{7}$. Another study demonstrated significantly better overall survival in patients aged 55-65 years with FLT3-negative, NPM1-positive AML (compared with other AML subtypes), although no significant difference was seen in patients aged over 65 years. ${ }^{8}$ Daver et al. found estimated 2 - and 5 -year overall survival rates of $37 \%$ and $28 \%$, respectively, for patients aged $\geq 65$ years with NPM1-positive AML treated with intensive chemotherapy. ${ }^{9}$

Hypomethylating agents, including decitabine and azacitidine, have proven effective in prolonging overall survival in AML patients not eligible for intensive chemotherapy, especially those with an unfavorable karyotype or mutational profile, and are approved in this situation in the European Union. ${ }^{10-13}$ The present work is, however, to our knowledge the first study of hypomethylating agents in NPM1-positive AML. The main message of our study is that, contrary to intensive chemotherapy, hypomethylating agents are unable to yield long-term survival when used in the first-line treatment of NPM1-positive AML. Our findings also suggest that when hypomethylating agents are used after failure of intensive chemotherapy (with or without allogeneic stem cell transplantation), the results are similar to those obtained in patients with other AML subtypes, without any prolonged responses unless the patients are eligible for subsequent allogeneic transplantation. ${ }^{14}$

The poor long-term results obtained in patients with NPM1-positive AML treated upfront with hypomethylat- ing agents, contrasting with the results of those given intensive chemotherapy, suggest that NPM1 status should be taken into account in addition to age and other parameters, such as karyotype, when making decisions concerning first-line treatment for elderly AML patients. The presence of an NPM1 mutation could contribute to physicians' decisions to administer intensive chemotherapy rather than a hypomethylating agent when there are no major contraindications to such chemotherapy.

Pedro Henrique Prata, ${ }^{1}$ Cécile Bally, ${ }^{1}$ Thomas Prebet, ${ }^{2}$ Christian Recher, ${ }^{3}$ Geoffroy Venton, ${ }^{4}$ Xavier Thomas, ${ }^{5}$ Emmanuel Raffoux, ${ }^{1}$ Arnaud Pigneux, ${ }^{6}$ Thomas Cluzeau, ${ }^{7}$ Judith Desoutter, Julie Gay,' Claude Preudhomme, ${ }^{10}$ Pierre Fenaux ${ }^{11}$ and Lionel Adès ${ }^{1}$

${ }^{\prime H}$ Hématologie Clinique, Hôpital Saint Louis, Paris, France; ${ }^{2}$ Yale Cancer Center, New Haven, CT, USA; ${ }^{3}$ Clinical Hematology, IUCT Oncopole, Toulouse University Hospital, France;

${ }^{4}$ Paoli-Calmettes Institute, Marseille, France; ${ }^{5}$ Lyon-Sud University Hospital, Pierre Bénite, France; ${ }^{6}$ Hematology Department, Centre Hospitalier Universitaire (CHU) de Bordeaux, Universités de Bordeaux, France; ${ }^{7} \mathrm{CHU}$ de Nice, France; ${ }^{8}$ Laboratoire d'Hématologie, CHRU Lille, Université de Lille, France; 'Service d'Hématologie, Centre Hospitalier de la Côte Basque, Bayonne, France; ${ }^{10}$ Centre de Biologie-Pathologie, Centre Hospitalier Universitaire de Lille, France and "Hopital Saint-Louis, Paris, France

Correspondence:lionel.ades@aphp.fr doi:10.3324/haematol.2018.189886

Information on authorship, contributions, and financial \& other disclosures was provided by the authors and is available with the online version of this article at www. haematologica.org.

\section{References}

1. Arber DA, Orazi A, Hasseriian R, et al. The 2016 revision to the World Health Organization classification of myeloid neoplasms and acute leukemia. Blood. 2017;127(20):2391-2406.

2. Thiede C, Koch S, Creutzig E, et al. Prevalence and prognostic impact of NPM1 mutations in 1485 adult patients with acute myeloid leukemia (AML). Blood. 2006;107(10):4011-4020.

3. Schnittger S, Schoch C, Kern W, et al. Nucleophosmin gene mutations are predictors of favorable prognosis in acute myelogenous leukemia with a normal karyotype. Blood. 2005;106(12):3733-3740.

4. Slovak ML, Kopecky KJ, Cassileth PA, et al. Karyotypic analysis predicts outcome of preremission and postremission therapy in adult acute myeloid leukemia: a Southwest Oncology Group/Eastern 
Cooperative Oncology Group study. Blood. 2000;96(13):4075-4084. 5. Cheson BD, Bennett JM, Kopecky KJ, et al. Revised recommendations of the International Working Group for diagnosis, standardization of response criteria, treatment outcomes, and reporting standards for therapeutic trials in acute myeloid leukemia. J Clin Oncol 2003;21(24):4642-4649.

6. Desoutter J, Gay J, Berthon C, et al. Molecular prognostic factors in acute myeloid leukemia receiving first-line therapy with azacitidine. Leukemia. 2016;30(6):1416-1418.

7. McGregor AK, Moulton D, Bown N, et al. Incidence and outcomes for adults diagnosed with acute myeloid leukemia in the north of England: a real world study. Leuk Lymphoma. 2016;57(7):1575-1584.

8. Ostronoff $\mathrm{F}$, Othus M, Lazenby M, et al. Prognostic significance of NPM1 mutations in the absence of FLT3-internal tandem duplication in older patients with acute myeloid leukemia: a SWOG and UK National Cancer Research Institute/Medical Research Council report. J Clin Oncol. 2015;33(10):1157-1164.

9. Daver N, Liu Dumlao T, Ravandi F, et al. Effect of NPM1 and FLT3 mutations on the outcomes of elderly patients with acute myeloid leukemia receiving standard chemotherapy. Clin Lymphoma
Myeloma Leuk. 2013;13(4):435-440.

10. Fenaux P, Mufti GJ, Hellstro E, et al. Azacitidine prolongs overall survival compared with conventional care regimens in elderly patients with low bone marrow blast count acute myeloid leukemia.. J Clin Oncol. 2010;28(4):562-569.

11. Dombret H, Seymour JF, Butrym A, et al. International phase 3 study of azacitidine vs conventional care regimens in older patients with newly diagnosed AML with > 30 \% blasts. Blood. 2015;126(3):291300.

12. Pleyer L, Döhner H, Dombret $\mathrm{H}$, et al. Azacitidine for front-line therapy of patients with AML: reproducible efficacy established by direct comparison of international phase 3 trial data with registry data from the Austrian azacitidine registry of the AGMT study group. Int J Mol Sci. 2017;18(2):415.

13. Welch JS, Petti AA, Miller CA, et al. TP53 and decitabine in acute myeloid leukemia and myelodysplastic syndromes. N Engl J Med. 2016;375(21):2023-2036.

14. Itzykson $R$, Thépot $S$, Berthon $C$, et al. Azacitidine for the treatment of relapsed and refractory AML in older patients. Leuk Res. 2015;39(2):124-130 